\title{
MCNPX'S Water Equivalent Thickness Simulation of Material with Different Density via Proton Beam Irradiation
}

\author{
M.A Khattak ${ }^{*}, 1$, Abdoulhdi. A. Borhana ${ }^{2}$, Lailatul Fitriyah A. Shafii ${ }^{3}$, , Rustam Khan ${ }^{4}$ \\ ${ }^{1}$ ARL Laboratory Services PTY Ltd. 13 61/55 Pine Rd, Yennora Sydney, NSW 216, Australia \\ ${ }^{2}$ Department of Mechanical Engineering, College of Engineering, Universiti Tenaga Nasional, 43000 Kajang, Selangor, Malaysia \\ ${ }^{2}$ Department of Mechanical Engineering, Faculty of Engineering Science and Technology, Sebha University, Libya. \\ ${ }^{3}$ Department of Nuclear Engineering, Faculty of Chemical Engineering, Univerisiti Teknologi Malaysia, 81310, Johor, Malaysia \\ ${ }^{4}$ Department of Nuclear Engineering, Pakistan Institute of Engineering and Applied Sciences (PIEAS), Islamabad,44000, Pakistan \\ *Corresponding author E-mail: adil@arllabservices.com.au
}

\begin{abstract}
The radiological thickness of materials and beam penetration range is often referred as the water equivalent thickness (WET). In the clinical application of radiotherapy it is mandatory to obtain a WET calculation with high accuracies to ensure the beam that penetrated the human tissues is capable to deliver high dose of radiation into the deep-rooted tumors and kill the malignant cancerous cell without any major damages to the healthy tissues. Nevertheless, the present method of calculation that is available needs either intensive numerical method or approximation techniques with unknown precision. Hence, the purpose of this research is to study the depth of proton beam irradiation penetration range of materials with arbitrary density \& elemental composition and modeled the water equivalent thickness (WET) calculation by using the Monte Carlo N Particle Transport Code Extension (MCNPX). There are several type of material with different density that are utilize in this project which are water phantom $\left(\rho=1.0 \mathrm{~g} \mathrm{~cm}^{-3}\right)$, PMMA $\left(\rho=1.19 \mathrm{~g} \mathrm{~cm}^{-3}\right)$ aluminum $(\rho=2.70 \mathrm{~g}$ $\mathrm{cm}^{-3}$ lead $\left(\rho=11.3 \mathrm{~g} \mathrm{~cm}^{-3}\right)$. The water phantom represent reference material whilst PMMA, Aluminum and Lead each represent low, medium and high density respectively. Based from the result produced in output file, Bragg curves for each material were reproduced, analyzed and compared with the Bragg curve of water phantom. The WET of water phantom was successfully modelled by using MCNPX. Apart from the short computing time, modelling WET via MCNPX was more efficient compare to analytical calculation
\end{abstract}

Keywords: Water equivalent thickness, MCNPX, proton therapy, Bragg peak

\section{Introduction}

Proton therapy is an example of particulate radiation therapy that differs from the typical electromagnetic radiation therapy such as photon in term of the unique peculiarity of the minimal dispersion as the charged beams penetrated the tissue and deposited its ionized energy at certain depth [1-2]. The proton deposition pattern known as the Bragg curve makes the radiation exposure emitted towards the neighbouring healthy tissues to be reduced [2]. In order to ensure that the beam that penetrated the human tissues is capable to deliver high dose of radiation into the deep-rooted tumors and kill the malignant cancerous cell without any major damages to the healthy tissues, the dose simulation requires computation of algorithm involving water equivalent thickness (WET) with high accuracies and capable to process the patient's tissue composition that is usually generated from the patient's computed tomographic (CT) images [3].

In proton therapy, water act as pseudo-tissue material in measuring the depth of beam penetration and dose deposited [4]. Water equivalent thickness (WET) or the 'penetration depth in liquid water' can be defined as the depth or range of beam penetration, as well as the thickness of components in proton beam path. In certain context it also can be referred to as water equivalent ratio (WER) which is the ratio of WET to material thickness [5]. Traditionally, the computation of WET in clinical application is done by calculating the range shift in immobilization device [5-6]. The present method of calcula- tion that is available needs either intensive numerical method or approximation techniques with undetermined accuracy.

The International Atomic Energy Agency (IAEA) suggested an estimation technique to proximate the WET that utilized ratio of continuous-slowing-down approximation (CDSA) for water, PMMA and polystyrene[8]. Whereas Newhauser et al purposed to compute WET of gold, stainless steel and titanium by using numerical method[9]. On the other hand, Zhang et al adduce to compute WET of lead, aluminum and PMMA by using simple deterministic method that was derived from Bragg-Kleeman rule (BK) and BetheBloch equation (BB) [10], [11]. Both studies done by Newhauser et al and Zhang et al revealed that the WET and WER calculation is dependent on proton energy intensity, density and thickness of materials. Nonetheless, these methods are time consuming and tedious which are not suitable for clinical application that required the fast and accurate results.

Researchers such as Fontenot et al and Lee et al utilized MCNPX to build their virtual proton facilities. Fontenot et al designed proton therapy nozzle that accentuate the double scattering foil method [12]. On the other hand, Lee et al produce a paper on the MCNPX simulation of proton dose distribution in CT phantoms [13]. Apart from that, Lee C.C et al published a paper on MCNPX of proton dose distribution in water phantom that study the fluence and dose distribution of proton in water phantom [14]. Monte Carlo (MC) technique is preferred among the researcher due to its efficient \& accurate WET computation competence to model the model of protons and secondary particles propagation [15-16]. Nevertheless, in 
this project the secondary particle produced during nuclear interaction is neglected.

In this study, the water equivalent thickness (WET) of each respective material that possess different density will be modeled by using MCNPX to simulate proton depth distribution. The WET of water phantom is used as reference material whereas lead, aluminum and PMMA is used to represent high, medium and low density materials. In addition, each of these target materials will be on the same dimension of $44 \mathrm{~cm} \mathrm{X} 44 \mathrm{~cm}$ X $44 \mathrm{~cm}$. A proton beam parallel along the z-axis with intensity of $175 \mathrm{MeV}$ (therapeutic proton beam) will be emitted onto the target materials. Based from the output file produced, Bragg curves for each respective material will be reproduced and analyzed.

\section{Methodology}

This study focused on the simulation of input file by using MCNPX. Fig.1 illustrate execution of MCNPX simulation. First and foremost, the WET measurement model was illustrated and translated into Input File that will later model via MCNPX Visual Editor (VISED). If the input file is failed to be modelled the error will be identified and once again run via VISED. If the input file is a success it will be simulated via MCNPX and an Output File will be printed.



Fig.1: The Flowchart of the Research Framework

\subsection{Water Equivalent Thickness (WET) Measurement Model}

The radiological thickness of materials and beam penetration range is often referred as the water equivalent thickness (WET). In the clinical application of radiotherapy, it is mandatory to obtain a WET calculation with high accuracies to ensure the beam that penetrated the human tissues is capable to deliver high dose of radiation into the deep-rooted tumours and kill the malignant cancerous cell without any major damages to the healthy tissues [17]. However, within a critical range of cumulative doses of 40-100 Gy when given with "conventional" fractionation of 1.8-2.0 Gy/fraction, small increases in dose may result in large increases in clinical impact. Fig. 2 shows the Water Equivalent Thickness measurement model constructed for this project.

In this project, the slab for the target material is defined with the dimension of $30 \mathrm{~cm} * 30 \mathrm{~cm} * 50 \mathrm{~cm}$ that comprised of 31 surfaces and 50 cells. These cells, known as scoring voxel is defined with the thickness of $1 \mathrm{~cm}$ along the z-axis and $30 \mathrm{~cm}$ for both the $\mathrm{x}$ and $\mathrm{y}$ axis to measure the water equivalent thickness of the given material. In addition, proton point source of mono-energetic beam with intensity of $175 \mathrm{MeV}$ is emitted towards the slab in the direction of $\mathrm{z}$ axis. Apart from the dimension of scoring voxel, in the WET measurement model, the source to surface distance is $80 \mathrm{~cm}$ which are comprises of air material slab and was defined between the proton source and the target material slab.

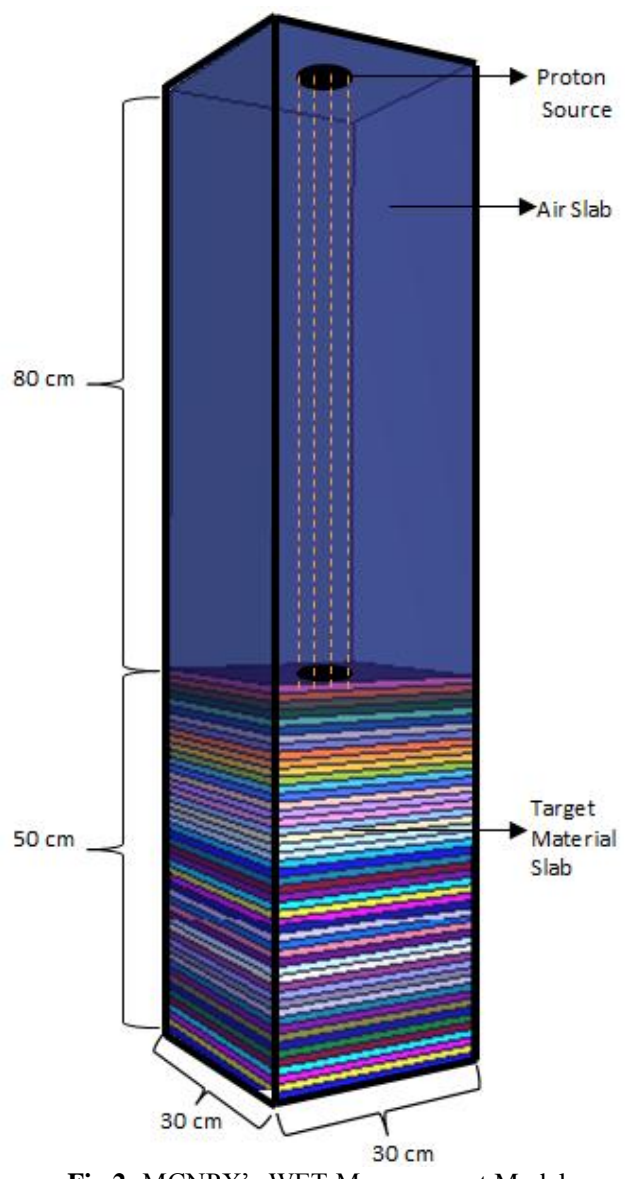

Fig.2: MCNPX's WET Measurement Model

Apart from that, in this project, the material defined are water, lead, aluminum, and PMMA each with density of $1 \mathrm{gcm}^{-3}, 11.3$ $\mathrm{gcm}^{-3}, 2.70 \mathrm{gcm}^{-3}$ and $1.19 \mathrm{gcm}^{-3}$ respectively. The scoring was performed using F6 tally that specifically calculate the energy deposition in $\mathrm{MeV} /$ gram and a one million $\left(10^{6}\right)$ of histories were simulated for variance reduction and to ensure that the tally passes all ten statistical test and relative error were less than $1 \%$. In addition, this project's modeling of WET only consider proton tracking and neglect the secondary particles that have been produced during proton nuclear interaction because in MCNPX, the secondary particle's energy is consider liberated at its point of generation.

\section{Preparation of Input File}

Input file is created by the user for the purpose to be read by the software. This file describe the problem's geometry specification, material's description, radiation source specification and the type of answers or tallies desired. The model illustrated in Fig. 2 is translated to codes and organised in the input file before they are run via MCNPX and Table 3.1 shows the general details of the input file. 
Table 1: Input File's General Details

\begin{tabular}{|l|c|}
\hline \multicolumn{1}{|c|}{ Subject } & Description \\
\hline Target materials and density & $\begin{array}{c}\text { Water Phantom }(\rho=1.0 \mathrm{gcm}-3) \\
\text { PMMA }(\rho=1.19 \mathrm{gcm}-3) \\
\text { Aluminium }(\rho=2.70 \mathrm{gcm}-3) \\
\text { Lead }(\rho=11.3 \mathrm{gcm}-3)\end{array}$ \\
\hline \multicolumn{1}{|c|}{ Target material dimension } & $30 \mathrm{~cm}^{*} 30 \mathrm{~cm}^{*} 50 \mathrm{~cm}^{*}$ \\
\hline Air material dimension & $30 \mathrm{~cm}^{*} 30 \mathrm{~cm}^{*} 80 \mathrm{~cm}^{*}$ \\
\hline SFD & $80 \mathrm{~cm}$ \\
\hline Total surfaces & 56 \\
\hline Total cell & 52 \\
\hline Source Specifications & Type: Proton point source \\
& Intensity: $175 \mathrm{MeV}$ \\
Position: $(0,0,130)$
\end{tabular}

The input file in MCNPX comprises of cell cards, surfaces card and data cards. The surface card is defined Cartesian coordinates system and represented functionally as $\mathrm{f}(\mathrm{x}, \mathrm{y}, \mathrm{z})=0$ whereas the cell card is defined by surfaces and Boolean logic. On the other hand, the data card describes the type of physical properties of materials used, specification of radiation source \& scoring of results (tallies) and the physics of radiation transport calculation to be performed [18].

\section{MCNPX Visual Editor Modelling}

The Visual Editor allows the user to easily set up and modify the view of the MCNPX geometry and to determine model information directly from the plot window [19]. It help user to interactively create an input file with the aid of the dynamic cross-sectional interface of the model (Fig. 3). Nonetheless, several alterations in the input file may be needed due to the difference in the density of material (water phantom, lead, aluminium and PMMA) and the accuracy of the results may vary from one to another.

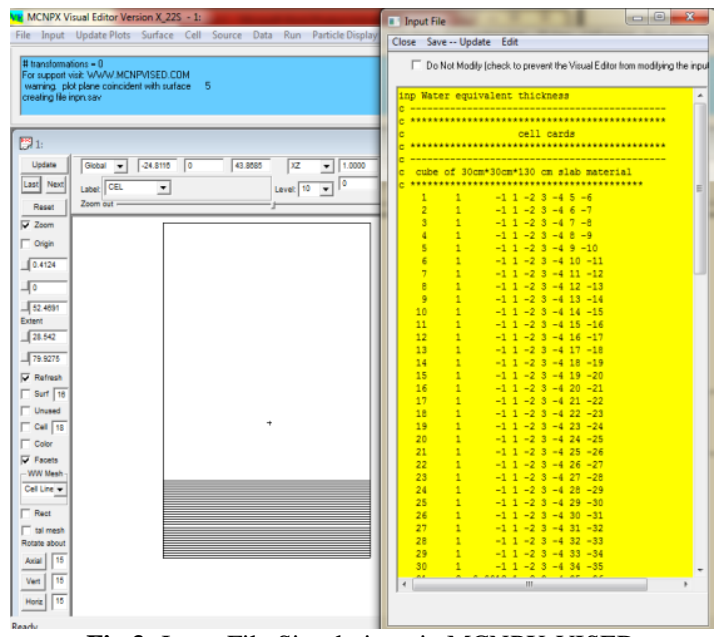

Fig.3: Input File Simulation via MCNPX-VISED

Before running the input file via MCNPX, the Input File is run via MCNPX Visual Editor (VISED) to ensure that the geometries that were done in the input file were coded correctly. This was since the process of creating an input file is both laborious and susceptible to error as it require strenuous description of geometry, tallies, sources and optimization parameter. Nonetheless, once the input file is modelled correctly via VISED, it will be simulated by using MCNPX to print an output file

\section{MCNPX's WET Simulation.}

Monte Carlo N-Particle Extension code (MCNPX) is multi-purpose code that specialized in finding proton depth dose and stopping power. Apart from that, it also applied in nuclear criticality calculations, radiation transport study, high energy dosimeter, charged particle tracking, neutron detection and designing of linear accelerator models and uses Fortran 90 Monte Carlo radiation transport computer code that can track 34 particle types, including four light ions, at nearly all energies [20].

MCNPX is an upgrade compared to its previous predecessor in term of the input file extension that enhances physics, source, tallies, graphics and infrastructure. Figure 3 shows the running process of MCNPX. MCNPX is the executable binary file and XSDIR is the cross-section directory. If the XSDIR is not available in the current directory, a DATAPATH need to be set. The secondary particles released from the beams are tracked from the birth to the ultimate absorption. Thus, the modeling of WET only consider proton tracking and neglect the secondary particles that have been produced during proton nuclear interaction.



Fig.4: Input File Simulation via MCNPX

This is due to the fact that in MCNPX, the secondary particle's energy is consider liberated at its point of generation. There are several execution commands in MCNPX which are ' $i$ ' to process an input file, ' $p$ ' to plot the geometry, ' $x$ ' to process cross section and ' $r$ ' to run the input file. Several alterations in the input file may be needed due to the difference in the thickness and density of material and the accuracy of the results may vary from one to another.The output desired in this project is the energy deposition that can be computed by using F6:n tally.

In addition to the tally information, the output file also comprised tables of standard summary information that aid user to understand the problem that was ran and provide insight into the physics of the problem and the adequacy of the Monte Carlo simulation. Apart from that, a detailed diagnostic prints for debugging is provided if errors occur when the problem is run. It also print the statistical relative error of each tally corresponding to its respective standard deviation.

Apart from the tally results, a comprehensive analysis is provided to assist in determining the validity of results. Ten pass/no pass checks are made for the user-selectable tally fluctuation chart (TFC) bin of each tally. Based from the output file generated, a Bragg curve of each respective materials will be plotted and analyse by using Microsoft Excel. The Bragg curves contain the information of energy deposition in $\mathrm{MeV} / \mathrm{g}$ for the $\mathrm{y}$-axis and proton penetration depth in $\mathrm{cm}$ for the z-axis.

\section{Results and Discussion}

This paper discusses the result of MCNPX's Water Equivalent Thickness simulation of water phantom, lead, aluminum and PMMA in proton beam irradiation. The results of these are discussed and compared based on its parameter and proton irradiation in water phantom is used as reference materials. In this project, the modelling of WET only considers proton tracking and neglects the secondary particles that have been produced during proton nuclear interaction. In MCNPX 2.7.0, the tracking of these secondary particles can be turned on or off.

If the tracking of any specific particle is turned off, its kinetic energy will be deposited locally and if we don't track secondary 
particle, its energy is assumed to be liberated at its point of generation. In a study done by Chao et al., 2015 conclude that the secondary particle only contributed about $0.1 \%$ of the dose but their simulation consumes about $60 \%$ of the computing time. Thus, it suggests that for radiation therapy purposes, the proton only simulation is optimal for both accuracy and efficiency. Nonetheless, the simulation for the secondary particles is only required for radiation safety issues of proton therapy.

The scoring for small voxels in the water phantom for the energy deposited at was done for different depth and is carried by using protons beam with energy of $175 \mathrm{MeV}$ (therapeutic proton beam) and $10^{6}$ numbers of histories. Fig 4 shows the results for the proton depth penetration of arbitrary materials. Basically, the Bragg curves for each respective material shows a similar energy deposi- tion pattern. In the entering region, protons have large kinetic energy and moves with large velocity. Thus, the dose deposited by the protons beams of each respective material remains almost the constant.

The small impulsive force between protons and medium electrons resulted the ionization of protons in the medium to be small. As the depth of protons penetration increases, the energy of the protons decreases and considerable time is available for protons to interact with medium electrons. Therefore, as the penetration depth increases, the amount of energy deposited increases. Nonetheless, eventually it gets neutralized by pick up of charge and hence shows the maximum energy deposition i.e. Bragg's peak. Once they've reach the maximum energy deposition, the energy deposited onwards (exit dose) is zero.

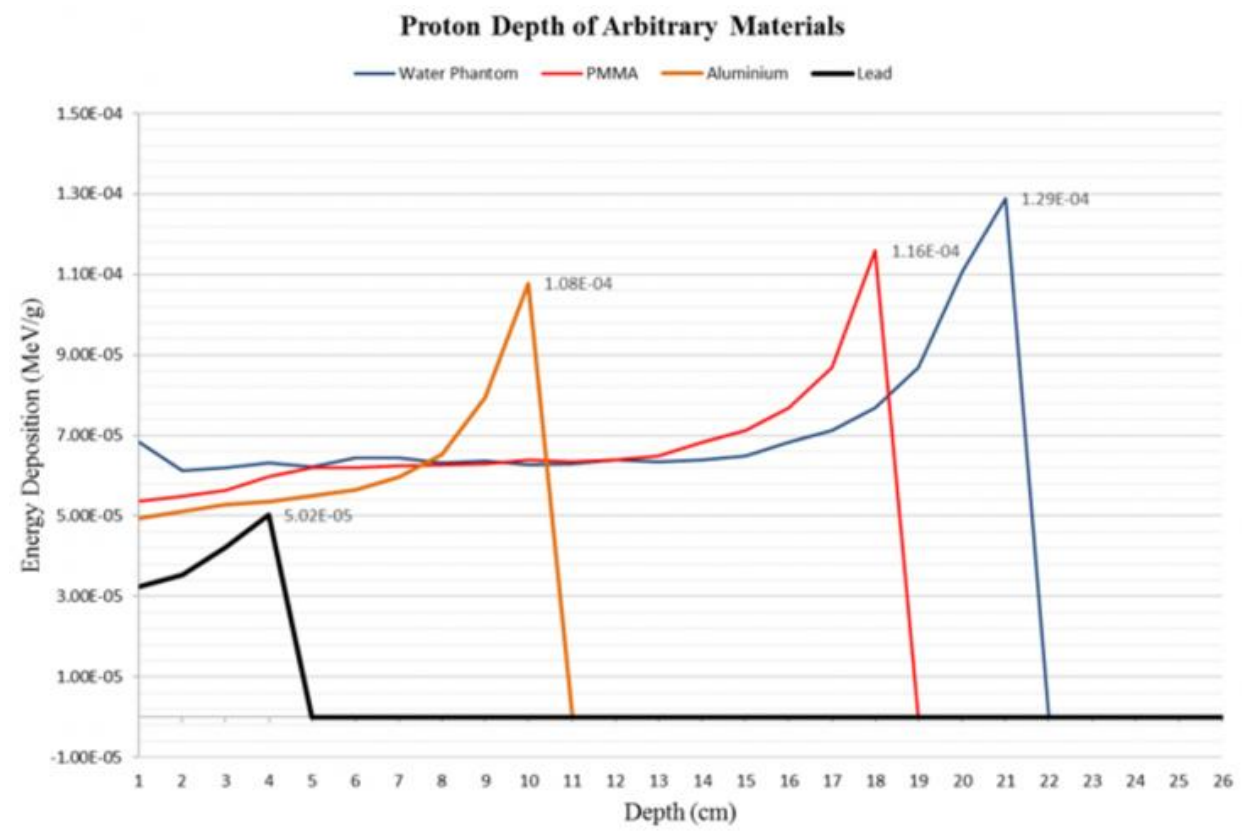

Fig.5: Results for Proton Depth of Arbitrary Materials.

Figure 5 shows that for the water phantom $\left(\rho=1.0 \mathrm{~g} \mathrm{~cm}^{-3}\right)$ that in the entering region, dose deposited by the protons remain almost constant in the first $16 \mathrm{~cm}$ of the depth and the energy deposited gradually increased from $6.82 \mathrm{E}-05$ (depth=16cm) to $1.11 \mathrm{E}-04$ (depth=20cm) $\mathrm{MeV} / \mathrm{g}$ and finally undergo energy explosion of $1.29 \mathrm{E}-04 \mathrm{MeV} / \mathrm{g}$ at the depth $21 \mathrm{~cm}$. As for PMMA $(\rho=1.19 \mathrm{~g}$ $\mathrm{cm}^{-3}$ ), the entering region is almost constant for the first $14 \mathrm{~cm}$ and the energy deposited gradually increase up to $8.68 \mathrm{E}-05 \mathrm{MeV} / \mathrm{g}$ at the depth of $17 \mathrm{~cm}$ before suddenly reach to an energy explosion of $1.16 \mathrm{E}-04 \mathrm{MeV} / \mathrm{g}$ at the depth of $18 \mathrm{~cm}$. On the other hand, for Aluminum $\left(\rho=2.70 \mathrm{~g} \mathrm{~cm}^{-3}\right)$, the energy deposited remain constant only for the first $6 \mathrm{~cm}$ before it gradually increases up to 7.94E-05 $\mathrm{MeV} / \mathrm{g}(\mathrm{depth}=9 \mathrm{~cm})$ and eventually rise to $1.08 \mathrm{E}-4 \mathrm{MeV} / \mathrm{g}$ at the depth of $10 \mathrm{~cm}$. Finally, for lead $\left(\rho=11.3 \mathrm{~g} \mathrm{~cm}^{-3}\right)$, the energy deposited only remain constant at the first $2 \mathrm{~cm}$ before it gradually increases to $4.22 \mathrm{E}-05 \mathrm{MeV} / \mathrm{g}$ at depth of $3 \mathrm{~cm}$ and the energy explosion of $5.02 \mathrm{E}-05 \mathrm{MeV} / \mathrm{g}$ occurred at the depth of $4 \mathrm{~cm}$ before it plunges to zero for the exit dose.

Based on Fig 5, the depth of proton penetration decreases as the material density increases. Although these respective materials is radiated by the proton source with the same intensity of $175 \mathrm{MeV}$, it can be observed that the energy deposited at the depth of $1 \mathrm{~cm}$ and the maximum energy deposited at the Bragg peak, differs for each respective materials. It can be summarized that as the density increases, the initial energy deposited the depth of $1 \mathrm{~cm}$ is smaller. In addition, the maximum energy deposited at the Bragg peak for each material also decreases as the density of the material increases.

\section{Conclusion}

In a conclusion, the WET of water phantom was successfully modelled by using MCNPX. Apart from the short computing time, modelling WET via MCNPX was more efficient compare to analytical calculation. This study also shows that as the relation of depth, density and beam penetration range where, as protons penetration depth increases, the energy of the protons decrease and a more time is available for protons to interact with medium electrons. Thus, as the depth increases, the energy deposited decreases. Apart from that, the energy deposited also decreases as the density decreases. Near the range of proton, it gets neutralized by pick up of charge and hence shows the maximum energy deposition i.e. Bragg's peak.

\section{Acknowledgment}

This work is supported by the Ministry of Higher Education (MOE) Malaysia through Fundamental Research Grant No. FRGS/1/2016/TK03/UNITEN /02/1 and FRGS-UTM4F420.

\section{References}

[1] W. D. Newhauser and R. Zhang, "The physics of proton therapy," Physics in Medicine and Biology, vol. 60, no. 8. pp. R155-R209, 2015.

[2] W. P. Levin, H. Kooy, J. S. Loeffler, and T. F. DeLaney, "Proton beam therapy," Br. J. Cancer, vol. 93, no. 8, pp. 849-854, 2005. 
[3] T. Rietzel, Eike; Geiß, Oliver; Schardt, Dieter; Voss, Bernd; Krämer, Michael; Haberer, "Correlation between CT number and WEPL for heavy charged particles," pp. 362-363, 2000.

[4] A. Kacperek, "Clinical Proton Dosimetry Part I: Beam Production, Beam Delivery and Measurement of Absorbed Dose (ICRU Report 59),” Phys. Med. Biol., vol. 45, no. 10, pp. 3123-3124, 2000.

[5] R. Zhang and W. D. Newhauser, "Calculation of water equivalent thickness of materials of arbitrary density, elemental composition and thickness in proton beam irradiation," vol. 27, no. 4, pp. 339 $351,2009$.

[6] F. Fellin, R. Righetto, G. Fava, D. Trevisan, D. Amelio, and P. Farace, "Water equivalent thickness of immobilization devices in proton therapy planning - Modelling at treatment planning and validation by measurements with a multi-layer ionization chamber," Phys. Medica, vol. 35, pp. 31-38, 2017.

[7] A. J. Wroe, A. Ghebremedhin, I. R. Gordon, R. W. Schulte, and J. D. Slater, "Water equivalent thickness analysis of immobilization devices for clinical implementation in proton therapy.," Technol. Cancer Res. Treat., vol. 13, no. 5, pp. 415-420, 2014.

[8] International Atomic Energy Agency, "Absorbed dose determination in external beam radiotherapy," 2000.

[9] W. Newhauser et al., "Monte Carlo simulations of the dosimetric impact of radiopaque fiducial markers for proton radiotherapy of the prostate," Phys. Med. Biol., vol. 52, no. 11, pp. 2937-2952, 2007.

[10] P. De Vera, I. Abril, and R. Garcia-Molina, "Water equivalent properties of materials commonly used in proton dosimetry," Appl. Radiat. Isot., vol. 83, pp. 122-127, 2014.

[11] R. Zhang, P. J. Taddel, M. M. Fitzek, and W. D. Newhauser, "Water equivalent thickness values of materials used in beams of protons, helium, carbon and iron ions," Phys Med Biol., vol. 49, no. 18, pp. 1841-1850, 2009.

[12] J. D. Fontenot, W. D. Newhauser, and U. Titt, "Design tools for proton therapy nozzles based on the double-scattering foil technique," Radiat. Prot. Dosimetry, vol. 116, no. 1-4, pp. 211-215 2005.

[13] C. C. Lee, Y. J. Lee, C. J. Tung, H. W. Cheng, and T. C. Chao, "MCNPX simulation of proton dose distribution in homogeneous and CT phantoms," Radiat. Phys. Chem., vol. 95, pp. 302-304, 2014.

[14] T.-C. Chao, C.-C. Lee, Y.-J. Lee, S.-K. Chen, B.-H. Chiang, and C.-J. Tung, "MCNPX simulation of proton dose distributions in a water phantom," Biomed. J., vol. 38, no. 5, p. 414, 2015.

[15] [15]J. S. Hendricks et al., "MCNPX extensions version 2.5.0," Work, p. 73, 2008.

[16] Oak Ridge National Laboratory, "MCNPX User's Manual Version 2.4.0," La-Cp-02-408, 2002.

[17] Y. Choi and Y. Kim, "Characteristics of neutrons and proton beams arising from two different beam nozzles," 2010.

[18] D. B. Pelowitz, "MCNPX user's manual," Los Alamos Natl. Lab. no. April, 2005.

[19] R. February, A. L. Schwarz, R. A. Schwarz, and L. L. Carter, "MCNP / MCNPX visual editor computer code manual," Los Alamos Natl. Lab., vol. 2008, 2008.

[20] F. B. Brown, "Fundamentals of Monte Carlo particle transport," Los Alamos Natl. Lab., vol. 836. 